\title{
Sick of Inequality? An Introduction to the Relationship between Inequality and Health
}

\author{
by Andreas Bergh, Therese Nilsson and Daniel Waldenström \\ Cheltenham (UK) and Northampton, MA: Edward Elgar, 2016 \\ ISBN 978-1-78536-420-4 \\ Hardcover \$99.95, 168 pp.
}

\section{Reviewed by Susan A. McDaniel University of Lethbridge}

This small book sets out a large agenda: to discuss the state of research on the relationship between inequality and health. Specifically, it seeks to examine the challenges in identifying the effect of inequality on health and how this effect might be robustly captured.

The book seeks to meet its objective in eight short chapters, following an introductory chapter and ending with an eight-page appendix. Chapters 2 (Measuring Health) and 3 (Measuring Inequality) outline and critique the usual ways that health and inequality are measured. Objective and subjective measures of health trends are contrasted and critiqued, noting that life expectancy has generally improved over time, while subjective health has remained flat-lined. Given this, the authors suggest that researchers might best use multiple measures of health. On measuring inequality, the authors take a similar interrogating approach, asking whether it is income or another distributional good that should be measured. They then outline the perils of assessing within and between country inequalities, and suggest caution and care in the selection of measures of both health and inequality. Each of these chapters has multiple evidentiary graphics.

Chapter 4 is more theoretical in examining the variety of ways research has indicated that economic inequality might affect health. Some pathways are psycho-social, while others focus more on monetary resources and still others rely on politics, crime, trust, or some other societal factors. The authors, in deconstructing explanatory frameworks, make the case that research must attend to a theoretical understanding of what underlying processes they are examining rather than treating the relationship of inequality to health as a given when an association is found. They further argue that inequality may not always have a detrimental health effect. The example they use is educational inequalities, which might provide incentives for enhancing education that benefits population health overall. They further suggest that there may be a threshold effect of inequality on health, in that the effect is only seen once inequality reaches a certain level. The threshold level may differ from society to society, or region to region. The threshold may be lower if inequality is perceived to be based on unfairness such as corruption or inheritance.

In chapter 5 , the focus shifts to the universal conundrum in the social sciences of correlation versus causation. Relying on correlational graphics-largely for Sweden, where two of the three authors are based-it becomes clear to the reader that scatterplots reveal no straightforward relation of inequality to health. Measures used matter to findings, as does geographic level. Further, of course, 
correlation, even if based on the best available and most appropriate measures and data, does not mean causality. Bergh et al. offer many examples of possible misinterpretations of associational relationships and further examples of possible reverse causality. They then work through an empirical exercise showing exactly how faulty interpretations can result when variables are relied upon without a clear theoretical model behind them, or without taking into consideration other factors that could affect the inequality/health relationship. This chapter could be an object lesson in being cautious in the use of statistical analyses. Chapter 7 adds further caution about reliance on aggregate data, as most studies of inequality and health tend to do.

Chapter 7 offers a concise and highly useful summary of the state of research on inequality and health. Although sizeable, this brief summary categorizes studies by measures used and by level of data, with a particular focus on those studies that combine individual and household data across regions and countries. In a simple graph (Figure 7.1), the authors reveal the immense variation in findings on the inequality/health relationship across studies. Two conclusions are reached, both with varying possible interpretations. First, the inequality effect seems stronger for subjective measures of health. And second, for objective health measures, very few find that income inequality is a significant health risk - a finding particularly clear in rich countries. Two additional critical observations emanate from this analysis, according to the authors: (1) Few studies offer conceptual frameworks of the inequality/health relationship; and (2) Very few studies offer a statistical approach that allows for causality to be assessed.

Chapter 8 offers an array of suggestions for measuring possible mechanisms at work in the relationship of inequality and health. And chapter 9 - relying on the sensational finding that Oscar winners live four years longer than other actors-shows the methodological flaw in this conclusion. Oscar winners often receive the award when they are older, and as demographers know well, life expectancy increases with years already lived. The chapter goes on to urge caution in interpretation of the purported inequality effect on health. They very clearly state that the effect cannot be dismissed as wrong, only that it should be examined with greater care and caution.

The lengthy appendix is a table with all 87 studies examined in the book listed by focal period, countries, measures of inequality and health, geographic level, findings and comments. This table is a valuable resource for any researcher who studies inequality and health, and should be well thumbed.

Sick of Inequality could be, as its subtitle suggests, an introduction to the relationship of inequality and health. However, most will find it daunting without a deep familiarity with the concepts and with the research methods used in the social sciences. This book would be more appropriate as a supplemental text in senior-level undergraduate or graduate-level courses in health sciences, sociology, demography, or political economy. It would also be useful for all researchers, including this reviewer, who purport to study the relation of inequality to health. Even broader than that, the book is an object lesson in the importance, in all the social sciences, of having solid theoretical frameworks guiding our empirical analyses. Without that, analyses-no matter how seemingly robust - may be subject to faulty interpretations, or worse. 